\title{
Photochromism in neuromorphic engineering
}

\author{
Pier Luigi Gentili ${ }^{1}$, Beatrice Bartolomei ${ }^{1}$, B. Mark Heron ${ }^{2}$, Jean-Claude Micheau ${ }^{3}$ \\ ${ }^{1}$ Department of Chemistry, Biology and Biotechnology, University of Perugia, Italy. \\ ${ }^{2}$ Department of Chemical Sciences, School of Applied Science, University of Huddersfield, UK \\ ${ }^{3}$ CNRS UMR 5623, IMRCP, University Toulouse III, Paul Sabatier, France
}

Artificial Intelligence (Al) is revolutionizing our societies. Al is applied in many fields: science, medicine, well-being, security, and economy [1]. There are two strategies to develop Al. One is by writing human-like intelligent programs running in computers or special-purpose hardware. The other is through neuromorphic engineering [2]. In neuromorphic engineering, surrogates of neurons are implemented through non-biological systems for neuro-prosthesis or brain-like computing machines. Surrogates of neurons can be implemented in hardware, i.e., in the solid phase, either rigid or flexible, depending on the materials used [3,4]. Alternatively, surrogates of neurons can be devised in wetware, i.e., in the liquid phase or even non-ideal medium, using specific solutions of non-linear chemical systems [5]. Finally, there is the possibility of using hybrid electrochemical systems [6]. In our group, we are mainly pursuing a wetware approach. We propose the use of peculiar chemical systems that can mimic the dynamics of real neurons in the liquid phase and outof-equilibrium conditions [7-13]. We usually exploit UV-visible radiation as signals. We study their optical communication, giving rise to feedback actions and emergent temporal synchronization phenomena, analogous to those shown by real neural networks $[9,10]$. In this contribution, we show how photochromic compounds are valuable in developing neuromorphic engineering. We demonstrate that single photochromic compounds are reasonable models of phasic excitable neurons [10]. Their wavelength-dependent photochemistry allows the implementation of neuromodulation [14]. Furthermore, photochromic systems can be models of pacemaker neurons [15] and extend human color vision to the UV $[16,17]$.

\section{References}

[1] S. J. Russell, P. Norvig, "Artificial Intelligence. A Modern Approach." (2009) Prentice-Hall, New Jersey.

[2] C. Mead, "Neuromorphic electronic systems." Proc IEEE 1990, 78, 1629-1636.

[3] J. L. Andrews, D. A. Santos, M. Meyyappan, R. S. Williams, S. Banerjee; "Building Brain-Inspired Logic Circuits from Dynamically Switchable Transition-Metal Oxides." Trends in Chem. 2019, 1, 711726.

[4] Y. Lee, T.-W. Lee; "Organic Synapses for Neuromorphic Electronics: From Brain-Inspired Computing to Sensorimotor Nervetronics." Acc. Chem. Res. 2019, 52, 964-974.

[5] P. L. Gentili, "Small steps towards the development of chemical artificial intelligent systems." RSC Adv 2013, 3, 25523-25549.

[6] Y. Liu, M. Sebek, F. Mori, I. Z. Kiss, "Synchronization of three electrochemical oscillators: From local to global coupling." Chaos, 2018, 28, 045104.

[7] P. L. Gentili, V. Horvath, V. K. Vanag, I. R. Epstein, "Belousov-Zhabotinsky" Chemical Neuron" as a Binary and Fuzzy Logic Processor." Int. J. Unconv. Comput. 2012, 8, 177-192. 
[8] V. Horvath, P. L. Gentili, V. K. Vanag, I. R. Epstein, "Pulse-Coupled Chemical Oscillators with Time Delay." Angew. Chem. Int. Ed. 2012, 51, 6878-6881.

[9] P. L. Gentili, M. S. Giubila, R. Germani, A. Romani, A. Nicoziani, A. Spalletti, B. M. Heron, “Optical Communication among Oscillatory Reactions and Photo-Excitable Systems: UV and Visible Radiation Can Synchronize Artificial Neuron Models." Angew. Chem. Int. Ed. 2017, 56, 7535-7540.

[10] P. L. Gentili, M. S. Giubila, R. Germani, B. M. Heron, “Photochromic and luminescent compounds as artificial neuron models." Dyes Pigm. 2018, 156, 149-159.

[11] P. L. Gentili, "The Fuzziness of the Molecular World and Its Perspectives." Molecules 2018, 23, 2074.

[12] P. L. Gentili; “Untangling Complex Systems: A Grand Challenge for Science." CRC Press, Taylor \& Francis Group, 2008, Boca Raton (FL, USA).

[13] P. L. Gentili, J. C. Micheau; "Light and Chemical Oscillations: review and perspectives" J. Photochem. Photobiol. C 2020, 43, 100321. doi.org/10.1016/j.jphotochemrev.2019.100321

[14] B. Bartolomei, B. M. Heron, P. L. Gentili; "A contribution to neuromorphic engineering: neuromodulation implemented through photochromic compounds maintained out of equilibrium by UV-visible radiation." Rend. Fis. Acc. Lincei 2020, 31, 39-52.

[15] P. L. Gentili, B. Bartolomei, J.-C. Micheau, "Light-driven artificial neuron models based on photoswitchable systems." Dyes Pigm. 2021, 187, 109086

[16] P. L. Gentili, A. L. Rightler, B. M. Heron, C. D. Gabbutt, "Extending human perception of electromagnetic radiation to the UV region through biologically inspired photochromic fuzzy logic (BIPFUL) systems." Chem. Commun. 2016, 52, 1474-1477.

[17] P. L. Gentili, A. L. Rightler, B. M. Heron, C. D. Gabbutt; “Discriminating between the UV-A, UV-B and UV-C regions by novel Biologically Inspired Photochromic Fuzzy Logic (BIPFUL) systems: A detailed comparative study." Dyes Pigm. 2016, 135, 169-176. 